\title{
Nécessité d'une gestion des résidus agricoles et agro-industriels à Kinshasa
}

\author{
Daniel-Bienvenu BANGALA MADA ${ }^{1 *}$, Pathy KANYANGA MPOY ${ }^{1}$, \\ Nadège KABAMBA NGOMBE ${ }^{2}$ et Thaddée MASIMANGO NDYANABO ${ }^{1}$ \\ ${ }^{1}$ Université de Kinshasa, Faculté des Sciences Agronomiques, \\ Département de Chimie et Industries Agricoles. 117 Kinshasa XI. Kinshasa, RDC. \\ ${ }^{2}$ Université de Kinshasa, Faculté de Pharmacie, Kinshasa, RDC. \\ *Auteur correspondant, E-mail : daniel_bangala@yahoo.com
}

\begin{abstract}
RÉSUMÉ
La pression humaine sur les ressources naturelles et la pollution environnementale, notamment par les résidus végétaux, constituent deux importantes conséquences du manque de politique structurée d'urbanisation et de traitement des déchets à Kinshasa. Une des pistes de solution à ce problème consiste à intégrer les acteurs qui sont à la base de ce problème. Dans les cas des résidus végétaux, il s'agit des producteurs agricoles et agroindustriels. Dans ce travail, une investigation a été menée auprès de trente producteurs agricoles et cinq agroindustriels localisés dans cinq communes de Kinshasa, dans le but d'évaluer le mode de gestion des résidus lignocellulosiques qu'ils génèrent par leurs activités. Aucun producteur agricole ni agro-industriel, ne dispose des outils d'estimation de la quantité des résidus qu'il génère ; les producteurs maraîchers utilisent presque tous les résidus de récolte pour la fertilisation des plates-bandes en vue de nouvelles cultures, tandis que les producteurs de riz ont un surplus de résidus. Les producteurs agro-industriels connaissent tous un problème d'élimination des résidus végétaux que leurs activités produisent.
\end{abstract}

(C) 2015 International Formulae Group. All rights reserved.

Mots clés : Environnement, pollution, lignocellulosiques, producteurs.

\section{Need for Management of Agricultural and Agro-industrial Residues in Kinshasa}

\begin{abstract}
The human strong need in natural resources and the environmental pollution, in particular by vegetable residues, represent two main consequences of the lack of structured urbanization policy and of the material waste processing in Kinshasa. One way of solutions to this problem consists in involving the persons who are on the basis of it. In the framework of vegetable residues, this concerns agricultural and agro-industrial producers. In this study, we aimed at evaluating the lignocellulosic residues management generated by thirty agricultural producers and five agro-industrials located in five municipalities of Kinshasa. Among agricultural and agro-industrials none have the use of tools necessary to estimate the amount of residues generated in their activities. Market gardeners use almost all residues of harvesting to fertilize plate bands for new cultivations while rice producers have surplus residues. All the agro-industrials experience a problem of elimination of vegetables residues generated by their activities.

(C) 2015 International Formulae Group. All rights reserved.
\end{abstract}

Keywords: Environment, pollution, lignocellulosic, producers. 


\section{INTRODUCTION}

La ville de Kinshasa, capitale de la République Démocratique du Congo (RDC), s'étend sur une superficie de $9.965 \mathrm{Km}^{2}$ (De Saint Moulin et Kalombo, 2005). Avec une population estimée à plus de dix millions d'habitants et en l'absence d'une politique d'urbanisation adéquate, cette ville subit deux phénomènes néfastes pour son épanouissement : la pression humaine sur les ressources naturelles et la pollution environnementale (Lelo, 2008).

En effet, à Kinshasa, l'accumulation des déchets de toute nature (matière plastique, épaves de véhicule, débris de végétaux, etc.) dans les lieux publiques et dans le cours d'eau cause une détérioration de l'environnement (Lelo, 2008), alors qu'il existe plusieurs possibilités de recyclage de ces différents types de résidus qu'ils soient biodégradables ou pas.

Les résidus végétaux qui sont aussi appelés résidus lignocellulosiques ou lignocellulose en raison de leur composition chimique dominée par la présence de trois constituants majeurs de la paroi cellulaire des végétaux, la cellulose, les hémicelluloses et la lignine (Klass, 2004; Ding and Himmel, 2006; Dermibas and Dermibas, 2007 ; Balat, 2011) sont issus de la végétation naturelle ou sauvage, de la production agricole, agroindustrielle, sylvicole et forestière non utilisée ainsi que d'une partie d'ordures ménagères solides (Hall et al., 1993; Dermibas and Dermibas, 2007).

Ceux-ci possèdent une très grande potentialité d'être recyclée en ce qu'ils peuvent servir soit de matière de base pour la production énergétique, soit de matière première pour la fabrication d'autres dérivées. La cellulose, de même que les hémicelluloses, en tant que glucides sont des sources d'énergie potentielle, soit par combustion directe ou indirectement pouvant servir à la production du biocarburant par fermentation alcoolique ou du biogaz par fermentation méthanogène (Müller and Trösch, 1986). Par ailleurs, la cellulose est une source de production du papier et d'autres produits (Thiébaud, 1995).

A Kinshasa, une valorisation des résidus végétaux présenterait donc le double avantage de réduire l'incidence de ces derniers dans la pollution environnementale et de fournir à la population de l'énergie bon marché ou de matériaux synthétiques.

Pour y arriver, la mise en œuvre d'une politique efficace de leur gestion doit dans un premier temps, intégrer les principaux acteurs à la base de leur production, c'est-à-dire, les producteurs agricoles et agro-industriels (Koopmans and Koppejan, 1997 ; Koyabizo, 2012). Ensuite, elle doit passer par une démarche de détermination de leur diversité, de leur caractérisation et de leur quantification (Koopmans and Koppejan, 1997 ; Jölli and Giljum, 2005; Cooper and Laing, 2007 ; Singh et al., 2008).

Pour évaluer le mode gestion des résidus lignocellulosiques générés par les producteurs agricoles, agroindustriels et les scieries de Kinshasa, une étude diagnostique a été menée dans la période entre janvier 2011 et décembre 2012.

Spécifiquement, ce travail a poursuivi les objectifs suivants :

- Identifier et quantifier les principaux résidus agricoles, agro-industriels produits dans quelques quartiers de Kinshasa à fortes activités agricoles et agro-industrielles ;

Evaluer le mode de gestion de ces sous-produits auprès de leurs principaux générateurs, c'est-à-dire, les producteurs agricoles et agroindustriels ;

- Déterminer les impacts éventuels positifs et négatifs liés à la présence de ces résidus ;

- Vérifier la capacité des producteurs d'utiliser les résidus générés par leurs activités

\section{MATERIEL ET METHODES}

Cette étude a été effectuée dans la ville 
de Kinshasa, une mégalopole de plus de 10.000.000 d'habitants, où les résidus sont produits abondamment et dont la gestion est un problème réel (Lelo, 2011; Koyabizo, 2012).

La méthodologie adoptée a été celle des investigations sur terrain sous forme d'enquête, accompagnée des observations visuelles. Les informations sur les principaux sites d'agriculture intense de Kinshasa ont été obtenues au niveau de la Direction de Production et de Protection Végétale du Ministère de l'Agriculture de la RDC. Les listes des grandes agro-industries et scieries de Kinshasa ont été fournies par la Cellule de Planification Industrielle du Ministère de l'Industrie, tandis que les scieries semiindustrielles ont été sélectionnées au hasard parmi celles qui sont disséminées dans les communes d'étude.

La ville de Kinshasa étant très vaste $\left(9.965 \mathrm{~km}^{2}\right)$, ce travail s'est limité dans cinq communes à forte production de ces résidus, sélectionnées sur base de leur accessibilité partant de l'Université de Kinshasa d'où l'étude a été lancée (Carte $n^{\circ} 1$ ).

Les communes concernées par cette enquête occupent la partie centrale et gauche de cette carte. Il s'agit de la commune de Lemba, de Limete (quartier industriel), de N'djili, de Kinsenso (quartier Ancien Combattant), et de Mont-Ngafula (cité de Kimwenza).

Après la collecte des données, le logiciel Excel a été utilisé pour la saisie et l'analyse des données. Dans ce cas, il s'agissait des statistiques descriptives (fréquence, valeurs limites, moyenne et écarttype).

Les investigations ont concerné les résidus lignocellulosiques issus de la production agricole, agro-industrielle et des scieries. Les fiches de questionnaires correspondant à chaque catégorie de résidu sont consignées dans les annexes et les principales questions qui ont fait l'objet de ces investigations sont résumées ci-dessous :

Questionnaire concernant les producteurs agricoles:

L'échantillonnage a été effectué au hasard et en fonction de la disponibilité des agriculteurs sur chaque site. Trente agriculteurs ont été questionnés dans la totalité dont onze à Limété Kingabwa TP, onze à Kisenso Anciens Combattants, quatre à N'djili Cecomaf et quatre à Mont-Ngafula Kimwenza.

La population d'enquête était constituée d'individus de deux sexes mais les hommes étaient plus nombreux (77\%). Parmi ces producteurs, la majorité était constituée des jeunes d'une moyenne d'âge de 34 ans. Concernant le niveau d'étude, la plus grande partie de la population d'enquête avait atteint le niveau secondaire mais sans le franchir (60\%). Il y avait 3\% d'analphabète, $20 \%$ s'étaient limités aux études primaires tandis que $17 \%$ avaient pu atteindre le niveau supérieur.

\section{Les questions posées aux agriculteurs concernaient La production}

Identité et activité principale du producteur; Les espèces végétales cultivées ; Nombre de plates-bandes emblavées par culture; Nombre de semis par mois ou par an ; Types et quantité de fertilisant par platebande; Nombre de récolte par mois ou par an ; Lieu de vente de la production.

\section{La gestion des résidus produits}

Existence des résidus de cultures après récolte; Usages des restes de cultures; Finalité des résidus de cultures non utilisés; Nuisance due à la présence des résidus ; Autres connaissances sur l'usage des résidus végétaux.

\section{Questionnaire concernant les agro-industries}

Chez cette catégorie, une attitude de réserve a été observée sur la livraison des informations jugées sensibles à cause de la concurrence. Il n'a pas été aisé d'obtenir des 
informations de la part des brasseries et de l'usine de torréfaction de café par crainte des agents espions de la sécurité et/ou des entreprises concurrentes.

Nos investigations ont concerné trois scieries dont l'une était industrielle (abrégée par SI), située dans la commune de Limété, quartier Kingabwa et les deux autres étaient semi-industrielles (abrégées par SSIa et SSIb), situées toutes dans la commune de Lemba. En plus des scieries, une usine de décorticage et de mouture de café (abrégée par UDMC) et une brasserie industrielle (abrégée par BI) situées toutes à Limeté Kingabwa ainsi qu'une usine de décorticage de riz (abrégée par UDR), située dans la commune de Ndjili ont été soumises à l'enquête.

Les questions posées concernaient

L'identité et les principales productions de l'entreprise

Catégorie ou type d'agro-industrie ; Localisation ; Principales productions ; Types et origine de la matière première

\section{La gestion des résidus}

Nature et quantité des résidus produits par jour (semaine, mois, an...) ; Proportion résidus sur production utilisable obtenue de la matière première; Usages réels des résidus ; Autres usages connus des résidus obtenus; Quantité de résidus non utilisés; Principales difficultés dans la gestion des résidus.

\section{Communes centrales}

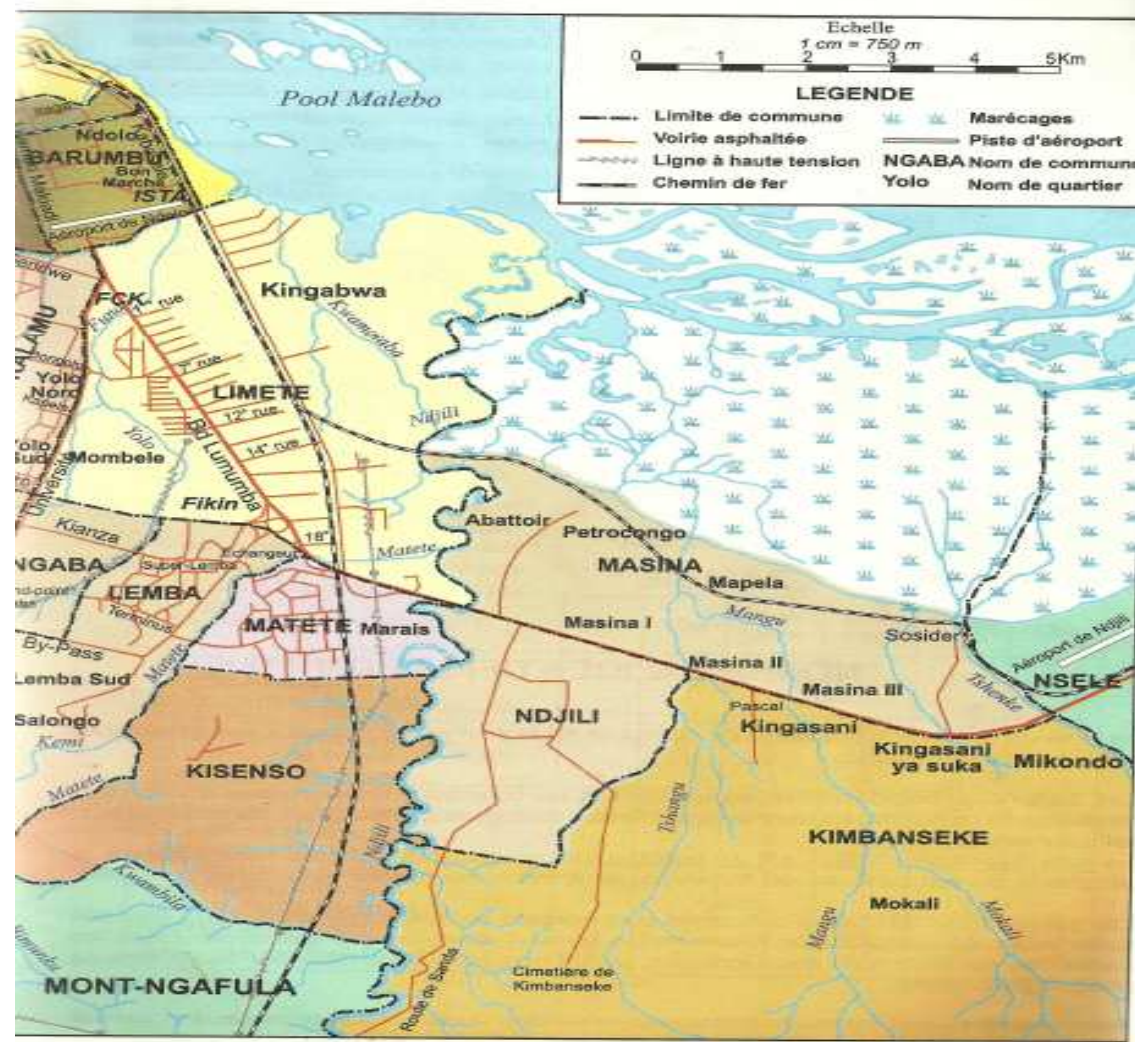

Figure 1 : Localisation des communes d'enquête (De Saint Moulin et Kalombo, 2005). 


\section{RESULTATS}

\section{Les producteurs agricoles}

\section{Production : Sites et types de cultures}

Théoriquement, les sites de culture sont aussi les premiers endroits où les résidus agricoles exercent leurs impacts après la récolte, tandis que les types de cultures pratiquées peuvent donner un renseignement sur la nature des résidus générés.

Les agriculteurs rencontrés aux sites des Anciens Combattant (abrégé par AC) et de Cecomaf (abrégé par CECO) sont tous producteurs des cultures maraîchères (amarantes, aubergines, gombo, oseilles, ail, oignons, etc.) tel que cela est révélé dans la Figure 1.

Au niveau de Kimwenza (abrégée par KIMW), en plus des cultures maraichères, certains agriculteurs produisent également du maïs et du manioc. Tandis que les agriculteurs questionnés au niveau de Kingabwa TP (abrégé par TP), en plus d'être producteurs des légumes, sont aussi producteurs de riz paddy et de maïs.

Dans les quatre sites confondus, $70 \%$ de la population d'enquête pratique le maraîchage. Cela laisse supposer qu'au moment de la récolte, les résidus générés par cette activité culturale devraient quantitativement être plus grande dans les quatre sites agricoles enquêtés. La section sur la gestion des résidus apportera la précision sur cette question.

A2. Nombre des plates bandes emblavées et cycle végétatif des cultures pratiques

Le nombre des plates bandes emblavées constitue une information importante sur la capacité des producteurs agricoles à générer des résidus végétaux sur un site donné. Théoriquement, il doit exister une proportionnalité entre ces deux variables.

Les nombres des plates-bandes emblavées sont variables suivant les producteurs questionnés et les types de culture qu'ils pratiquent. La Figure 2 renseigne sur la répartition des plates bandes par site d'enquête :

La figure ci-dessus nous informe que le site des TP renferme les plus petits producteurs de cette enquête (une à vingt plates-bandes), tandis que les producteurs emblavant jusqu'au delà de quarante platesbandes se retrouvent dans les trois autres sites. Théoriquement, la quantité des résidus que ces derniers génèrent devrait être tout aussi importante.

La connaissance du cycle végétatif est importante pour déterminer la période où une culture donnée est susceptible de générer des résidus. Ce moment arrive à la fin du cycle végétatif, au cours de la récolte.

Selon les producteurs interrogés, à part le gombo dont le cycle végétatif varie de trois à quatre mois, les cultures maraîchères ont un cycle mensuel, c'est-à-dire, qu'elles sont cultivées et récoltées douze fois par an.

Le maïs est cultivé deux fois l'an (septembre et mars) pour être récolté respectivement en février et en juillet. Quant à la culture du manioc, elle est faite en septembre et en février-mars pour être récoltée douze mois plus tard. Le riz est cultivé une fois par an et est récolté en mai et juin.

Les renseignements ci-dessus nous amènent à suggérer les hypothèses suivantes :

- Sur les sites de production des cultures maraîchères enquêtés, il y aurait des résidus de cultures maraîchères chaque mois de l'année, au moment de leur récolte ;

- Les résidus de manioc, par contre, seraient disponibles en septembre, février et mars ;

- Les résidus de maïs seraient plus disponibles aux mois de février et de juillet ;

- Les résidus issus de la riziculture seraient plutôt présents aux mois de mai, juin et parfois juillet.

\section{Utilisation d'engrais}

L'information concernant l'utilisation 
d'engrais peut être pertinente en ce que les agriculteurs de Kinshasa utilisent les restes de cultures comme engrais organiques. Cette donnée renseigne sur la capacité de ces producteurs agricoles d'utiliser partiellement ou totalement les résidus agricoles qu'ils génèrent.

Deux types d'engrais sont utilisés par tous les producteurs : les engrais organiques et les engrais chimiques.

\section{Engrais organiques}

Pratiquement tous les producteurs questionnés utilisent directement les restes de la culture précédente pour fertiliser les nouvelles plates-bandes emblavées. D'autres y ajoutent des herbes mortes, du compost qu'ils fabriquent ou achètent, du lisier de porcs et de la fiente de poules.

\section{Engrais chimiques}

Les agriculteurs interrogés utilisent trois types d'engrais chimiques (Figure 3). Il s'agit du NPK, de l'urée et de l'hydrogenophosphate d'ammonium (qu'ils appellent ammoniaque). Selon les producteurs questionnés, ils recourent aux engrais chimiques à cause de l'insuffisance d'engrais organiques dont ils ont besoin pour leur activité culturale.

A la lumière de cette figure, $70 \%$ des producteurs agricoles soumis à cette enquête utilisent les engrais chimiques pour suppléer à l'insuffisance d'engrais organiques. Le NPK est utilisé par $17 \%$ des producteurs maraîchers, l'urée par $61 \%$ des producteurs maraîchers et $25 \%$ des producteurs de riz tandis que le DAP est utilisé par $8 \%$ des producteurs maraîchers.

\section{Lieu de vente de la récolte}

L'information relative au lieu de vente de la récolte permet d'étendre le diagnostique sur la production des résidus agricoles au delà du lieu et du moment de la récolte. En effet, par l'opération de vente, la production végétale est exportée hors du champ et peut occasionner la production des résidus dans un environnement qui n'est pas sous le contrôle du producteur.

La préférence des enquêtés en ce qui concerne les lieux de vente de leur récolte, se présente comme suit (Figure 4) :

Il ressort de cette figure que $73 \%$ d'enquêtés vendent leur production à partir du champ, 20\% parmi eux transportent leur production au marché, tandis que 7\% emportent leur production à la décortiqueuse. Ces derniers produisent du riz cargo.

Le mode de vente varie d'une culture à une autre. En maraîchage, le producteur laisse à l'acheteur le soin de récolter les plantes arrivées à maturité. Généralement, celui-ci emporte toute la production qu'il achète, en laissant très peu de résidus. Les riziculteurs abandonnent leurs récoltes sur le champ jusqu'à ce qu'elles sèchent. Ensuite, ils battent les tiges sèches de riz pour en récupérer le riz cargo. Ce dernier est directement vendu ou alors transporté à la décortiqueuse. Les producteurs de maïs et de manioc vendent leurs productions directement après la récolte.

Lorsque l'agriculteur vend sa production, il peut être confronté à la gestion des résidus issus de son travail agricole. Les lignes qui suivent donnent les informations relatives à la manière dont la population d'enquête arrive à gérer les résidus générés par son travail agricole.

\section{Gestion des résidus de culture}

Tous les producteurs questionnés reconnaissent qu'il reste des résidus au champ après leur récolte, cependant aucun parmi eux n'a des précisions sur la quantité de ces résidus.

Concernant l'usage de ces résidus, en culture maraîchère, $82 \%$ des producteurs questionnés recyclent complètement leurs résidus en les utilisant au cours de la fertilisation en vue des nouvelles cultures. Néanmoins, $8 \%$ parmi ceux-ci, 
essentiellement des producteurs de Gombo, attestent qu'il reste encore des résidus qui sont écartés ou brûlés. Tous les producteurs de riz et $20 \%$ des producteurs de maïs avouent également l'existence des résidus inutilisés après la récolte. Ces derniers sont abandonnés à côté des champs ou alors brûlés.

Quelques-uns parmi ces producteurs reconnaissent que les résidus qu'ils abandonnent ou brûlent à côté de leurs champs ont un impact négatif sur l'environnement. Ils estiment que ces résidus devraient plutôt être affectés à l'alimentation animale.

\section{Les producteurs agro-industriels Matière première et Production}

L'UDMC utilise le café parche (variété robusta) comme matière première. Toute la production vient de l'intérieur de la RDC, notamment des provinces de l'Equateur, du Bas-Congo, de Bandundu et du KasaïOriental. Le rythme de réception de cette matière est variable au cours de l'année, toutefois, c'est aux mois de mai, juin, septembre, octobre et novembre que l'offre est la plus importante

La principale production de cette agroindustrie est le café décortiqué, torréfié et moulu. Ce dernier est conditionné dans des petits sacs en plastiques de $25 \mathrm{~g}$ et vendu dans les boutiques et marchés de Kinshasa. L'UDMC n'a fourni aucune information sur la production journalière ou mensuelle de leur café commercialisable.

La BI utilise le malt ou l'orge germé et le riz décortiqué importés de l'étranger comme principales matières premières. Outre les matières premières, d'autres constituants mineurs sont aussi ajoutés à la fabrication de la bière. La fréquence de leur réception et la quantité du produit fini obtenu en fonction du temps n'ont pas été révélées.

L'UDR reçoit sa matière première, le riz paddy, provenant de différents producteurs de Kinshasa. Sa fréquence de réception qui n'est pas enregistrée est variable au cours de l'année. Néanmoins, l'usine est ravitaillée en grande quantité autour du mois de mai.

La principale production de l'UDR de Ndjili est le riz décortiqué. La production journalière de ce dernier oscille entre $500 \mathrm{~kg}$ et $8000 \mathrm{~kg}$ par jour.

La SI reçoit comme matière première les grumes de bois (Kambala ou Milicia excelsa, Afrombosa ou Pericopsis elata, bois rouge ou lifaki qui est une Meliacée, bois noir ou Milletia laurentii, titola, iroko ou Milicia excelsa et autres) provenant de la province Orientale, de Bandundu, de l'Equateur et du Kasaï-Occidental. Le rythme de réception de la matière première est variable mais, en moyenne, elle est de 250 à $300 \mathrm{~m}^{3}$ de grumes par mois.

La SI produit du bois scié à partir des grumes qu'elle reçoit, selon la forme et les dimensions des clients. La production journalière est variable.

La SSIa se charge de raboter, réduire de dimensions ou donner une forme particulière à tout objet en bois selon la demande de sa clientèle. Cette dernière est constituée des menuiseries et des particuliers localisés dans ses environs. La fréquence de réception est très variable et quotidiennement fluctue entre 2 et 50 pièces.

La SSIb est destinée à approvisionner une boulangerie locale en lattes de bois. Ces dernières servent de combustibles dans les fours utilisés pour la cuisson des pains. Elle reçoit des fragments de bois de coffrage provenant de différentes scieries industrielles de Kinshasa à un rythme hebdomadaire. La livraison de ces morceaux de bois est assurée par un camion de marque MAN d'une capacité de $7 \mathrm{~m}^{3}$. La production quotidienne des buchers est d'environ 50 articles en 8 heures de service.

\section{La gestion des résidus}

Types et quantités journalières de résidus produits

L'UDMC: le café parche produit comme principal résidu la parche dont la 
quantité moyenne hebdomadaire est de l'ordre de 24 sacs cylindriques en polypropylène de $110 \mathrm{~cm}$ de hauteur et $45 \mathrm{~cm}$ de diamètre. Ces derniers sont stockés à côté de l'entrée principale de l'usine. Bien que n'ayant pas été pesés au cours de l'enquête, nous estimons que ces sacs remplis de parches doivent avoir chacun plus de $50 \mathrm{~kg}$. La quantité de café engendrant cette quantité de résidus n'a pas été révélée.

Ces résidus de café sont à la base de la production des moustiques et des eaux stagnantes après les pluies. Pour faire face à la difficulté engendrée par la présence de ces résidus, une importante quantité est offerte gratuitement aux femmes productrices des légumes, tandis que le reste, de 10 à 12 sacs par semaine, est éliminé dans une rivière passant à côté de cette entreprise (Photo $\left.{ }^{\circ} 1\right)$.

La BI : la drêche constitue le principal résidu solide engendrée par la brasserie. La quantité quotidienne de drêche produite par la BI ne nous a pas été donnée. Toutefois, concernant le ratio résidu/production, il nous a été signalé qu'environ $17 \%$ de drêche sont produites par la quantité d'orge (non précisée) donnant un brassin de 580 hectolitres.

Concernant son utilisation, la brasserie vend à très vil prix (50 CDF par chargement d'un camion MAN de 12 à 17 tonnes) la drêche aux éleveurs de porcs et de poissons, ainsi qu'aux producteurs de légumes. Pour éviter toute nuisance pouvant être causée par la quantité de drêche non vendue, celle-ci est déversée dans le fleuve passant à proximité de la BI.

L'UDR : Les sons et balles sont les principaux résidus issus du décorticage du riz. L'UDR produit quotidiennement une quantité des balles de riz variant entre 2 et 10 sacs en polypropylène (de même type que ceux utilisés pour les parches de café), en période de faible activité et entre 50 et 100 sacs lorsqu'il y a abondance de la clientèle aux mois de juin, juillet et août. Le ratio résidus/produit fini du décorticage est de 30 à $40 \%$ (son et balles de riz), il est de 17 à $25 \%$ pour les balles seules.

La SI : la SI génère trois types de résidus : la sciure, les lattes et les dos de bois. La quantité moyenne de ces résidus produits quotidiennement est d'environ 30 sacs de sciure (sacs de même type que ceux utilisés pour les parches de café et les balles de riz), 40 à 50 lattes en bois des tailles variables, et une quantité non déterminée de dos de bois. $100 \mathrm{~kg}$ de grumes génèrent, après leur traitement, environ $15 \mathrm{~kg}$ de résidus. Ils sont utilisés pour la fabrication des tabourets, des cercueils et distribués aux mamans maraîchères. La portion de la sciure non évacuée est entassée à côté du mur de la scierie (photo $\mathrm{n}^{\circ} 2$ ). Quand il pleut, il se forme à cet endroit une abondante boue.

La SSIa : cette scierie produit quotidiennement une quantité de 3 à 10 sacs en polypropylène, de même type que ceux cités précédemment (photo ${ }^{\circ} 3$ ), de sciure et copeaux de bois. Le ratio résidus sur produit utile n'est pas calculé. Généralement, ces résidus sont donnés gratuitement aux mamans des environs qui les utilisent comme fertilisants organiques, combustibles et litières dans l'élevage des volailles.

En dépit de cela, la SSIa éprouve encore de difficultés pour se débarrasser totalement de tous les sacs de résidus stockés dans son enceinte comme cela est révélé dans la photo ci-dessus. Il s'ensuit l'encombrement de l'espace, la formation des eaux stagnantes et le pullulement des moustiques.

La SSIb : produits quotidiennement environ 3 sacs de sciure et copeaux de bois. Le ratio quantité des résidus sur quantité de production utile n'est pas connu. Par ailleurs, ces résidus qui sont à la base de la formation des boues après les pluies et du pullulement des moustiques, sont d'une manière générale vendus aux femmes des quartiers environnant cette scierie. 
D. B. BANGALA MADA et al. / Int. J. Biol. Chem. Sci. 9(4): 2234-2248, 2015

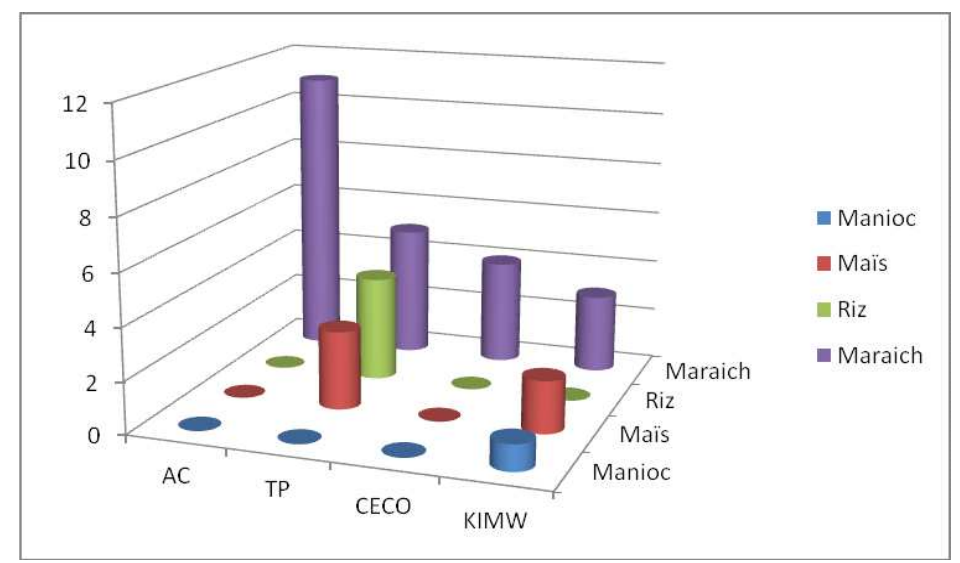

Figure 1 : Répartition des cultures par site d'enquête.

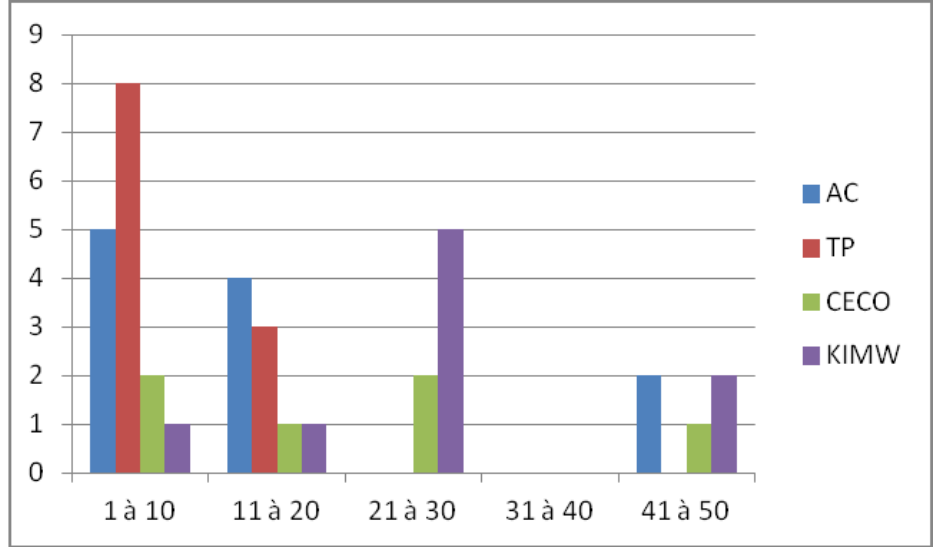

Figure 2 : nombre de plate-bande par site d'enquête.

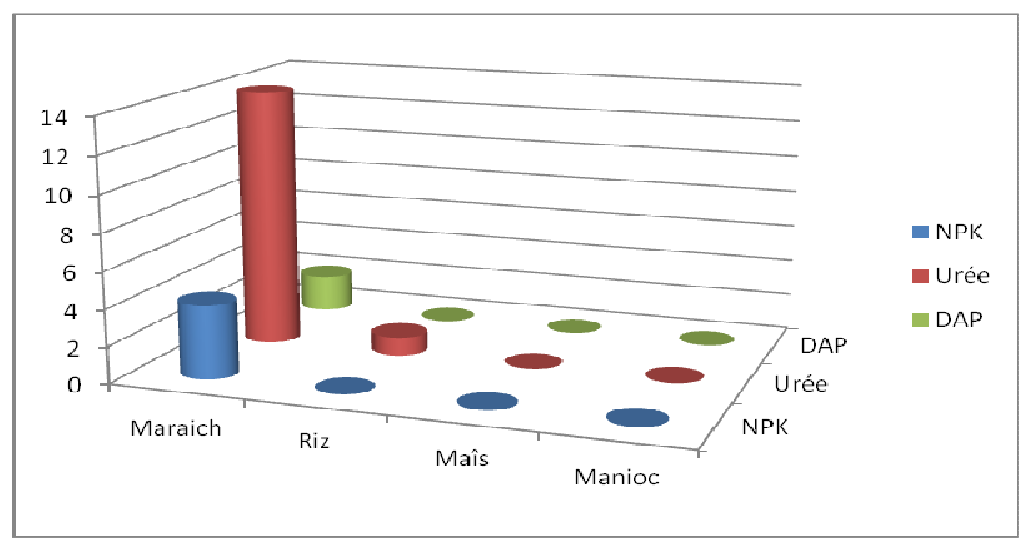

Figure 3 : Types d'engrais chimiques utilisés pour la production agricole dans les sites d'enquête. $\mathrm{DAP}=$ diammonium phosphate ou hydrogenophosphate d'ammonium. 
D. B. BANGALA MADA et al. / Int. J. Biol. Chem. Sci. 9(4): 2234-2248, 2015

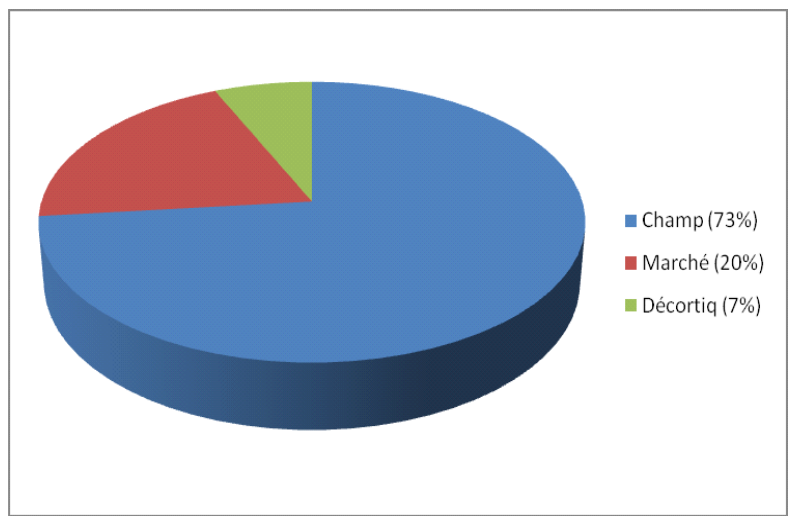

Figure 4 : Lieux de vente de la production.

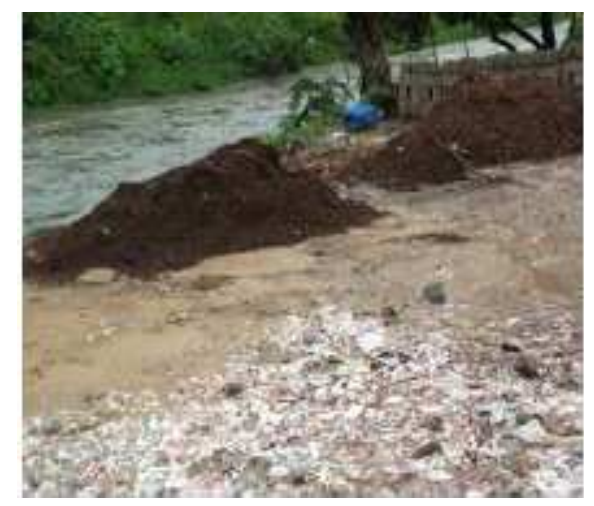

Photo 1 : Parche de café entassée à côté d'une rivière à Kinshasa Kingabwa.

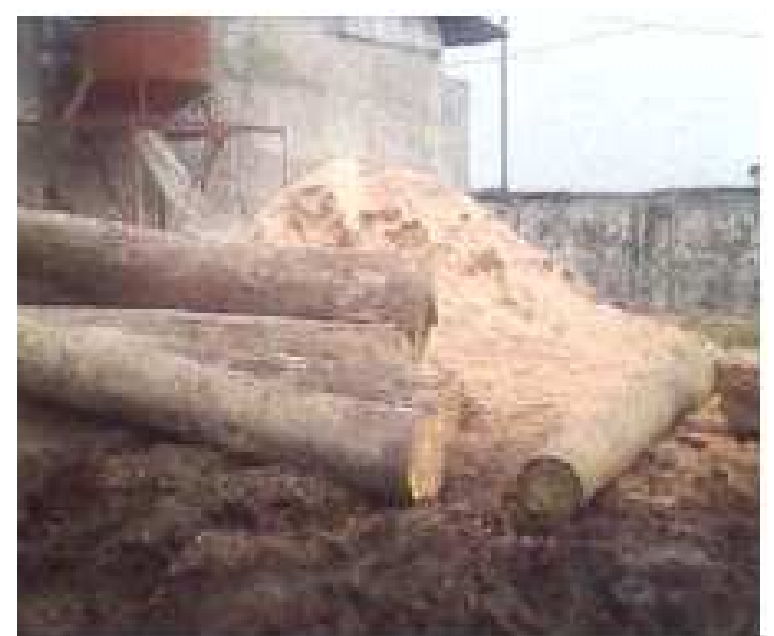

Photo 2 : Sciure et grumes de bois jonchant le sol à côté du mur de la scierie SI à Kinshasa Kingabwa. 


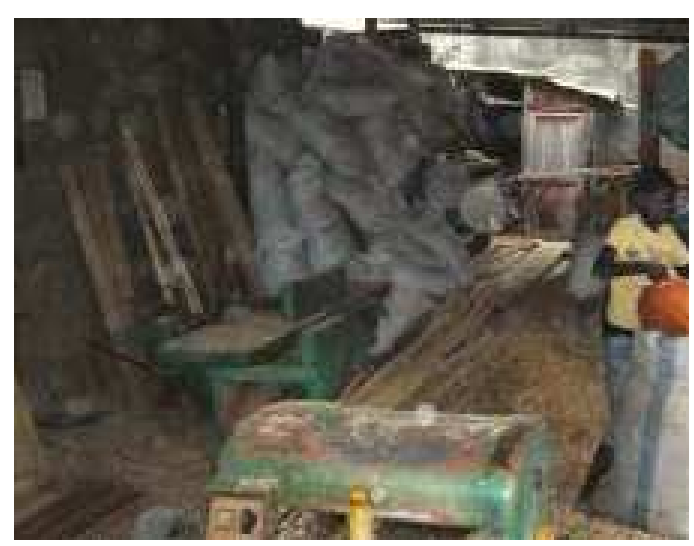

Photo 3 : Sacs contenant de la sciure de bois dans la scierie SSIa à Kinshasa Lemba.

\section{DISCUSSION}

Au cours de cette enquête, il a été noté qu'en culture légumière, il y a régulièrement production des résidus au moment des récoltes. Cependant, en dépit du nombre des plates bandes qui donnent ces légumes et du caractère mensuel de la production, la quantité de ces résidus demeure insuffisante pour couvrir le besoin en fertilisants organiques requis pour les prochaines cultures. Le même constat a été fait chez la majorité des producteurs de maïs. Cependant, tous les producteurs de riz cargo disposent des résidus non quantifiés, inutilisables après la récolte.

Mulaji (2011) estime que la carence en résidus végétaux nécessaires à la fertilisation organique du sol concerne la pratique agricole dans toute la ville de Kinshasa. Cette situation serait principalement due, estime-t-il, à la nature altérée, acide et pauvre en nutriments des sols de cette ville. Pour faire face à cette situation de carence, il recommande la valorisation de la matière organique des déchets solides ménagers par leur recyclage en agriculture sous forme de compost.

La question de recyclage et de valorisation des résidus agricoles est assez délicate et préoccupe la communauté scientifique même au delà de nos frontières nationales. Au Sénégal, en guise d'illustration, le groupe Ingenosahel (1998) entrepris une enquête sur la disponibilité et la valorisation des résidus agricoles et agroindustriel. Sur base des résultats obtenus, ce groupe recommanda le maintien en terre d'une bonne portion des résidus agricoles pour la préservation de la fertilité des sols et la prévention des érosions dans ce pays. Parmi ces résidus figurent les pailles de mais et de riz.

En tenant compte des résultats de la présente enquête auprès des producteurs agricoles, des recommandations formulées par Mulaji (2011) et le groupe Ingenosahel (1998), le scénario envisageable pour la valorisation des résidus agricoles à Kinshasa consisterait à mettre en place une stratégie basée sur la localisation des sites où ces résidus sont excédentaires, l'identification et la quantification de ces derniers, la détermination des besoins chez les producteurs en carence et la mise en œuvre des moyens d'approvisionnement de ceux-ci en engrais organiques.

En analysant les informations récoltées auprès des agro-industries concernées par cette enquête, les observations suivantes se dégagent :

Concernant la quantification, parmi les agro-industries enquêtées, l'UDMC, l'UDR, la SI, la SSIa et la SSIb ne procèdent pas rigoureusement à la quantification de leurs 
résidus mais elles disposent des données qui peuvent servir à déterminer, plus ou moins grossièrement, la quantité des résidus que leurs activités génèrent. Ces données peuvent constituer un point de départ pour la mise en place d'une filière de leur valorisation. Par contre, il est très difficile de tenter cette estimation avec les données obtenues de la BI.

Quand à l'usage de ces résidus, aucune de ces entreprises ne semble mettre en ouvre une technologie permettant de créer une valeur ajoutée à ces sous-produits. Ce qui est noté, c'est un effort énorme que ses entreprises fournissent pour de se débarrasser de leurs résidus, à cause de toute sorte de nuisances qu'ils causent. Il s'agit de la pullulation des moustiques, la création des eaux stagnantes et d'autres.

Toutes ces agro-industries évacuent leurs résidus en les fournissant gratuitement ou les vendant à vil prix aux producteurs maraîchers ou aux éleveurs de volailles. En dépit de cela, il reste généralement une quantité résiduelle qui est déversée dans les cours d'eau, fleuve ou rivière, passant à proximité de certaines de ces entreprises.

En considérant les deux catégories de producteurs ayant fait l'objet de cette enquête, les agriculteurs et les agroindustriels, nous observons que les résidus végétaux issus de la riziculture et des agro-industries constituent, d'une part, une certaine nuisance pour leurs producteurs, tandis que d'un autre côté, les producteurs maraîchers en ont besoin comme engrais organiques. Nous estimons que cette situation est en grande partie due à l'absence d'une politique provinciale de valorisation des résidus végétaux (Lelo, 2008; Holy et al., 2013 ; Holy et al., 2014).

En effet, à l'heure actuelle, contrairement à la situation que nous vivons en RDC, les résidus végétaux ne sont plus considérés comme nuisance dans plusieurs pays d'Afrique et d'autres continents. Plusieurs états s'adonnent à l'élaboration des statistiques annuelles de tous les types de résidus agricoles et agro-industriels produits dans l'ensemble de leurs territoires nationaux.
Ces informations leur permettent de planifier des politiques de valorisation de ces sousproduits (Amoo-Gottfried and Hall, 1999; McKendry, 2002 ; Jingura and Matengaifa, 2008 ; Sing et al., 2008).

Le raisonnement moderne qui soustend la mise en œuvre des méthodes de valorisation de la lignocellulose est donné par Koopmans \& Koppejan (1997) et Thioune (2009). Il se résume ainsi : «les résidus agricoles, agro-industriels et la biomasse inutilisée, longtemps considérés comme une menace pour l'environnement et la santé des populations, qu'il fallait nécessairement détruire, sont, aujourd'hui, considérés comme une richesse énergétique, en tant que sources d'énergies alternatives aux combustibles traditionnels». En plus d'usage comme combustible, ces résidus peuvent aussi servir comme substrat pour la production des champignons comestibles, la fabrication du compost et d'autres produits biobasés (Sivrikaya and Peker, 1999 ; Carmen, 2010 ; Binod et al., 2010).

Cette enquête s'est limitée à la production et la gestion des résidus végétaux générés au niveau des champs et des agroindustries dans quelques communes de Kinshasa. Concernant la production et gestion de ces résidus dans les circuits de commercialisation au niveau des marchés et dans les ménages consommateurs finaux, les travaux de quelques auteurs qui se sont intéressés à la gestion des déchets solides produits dans la ville de Kinshasa peuvent être consultés (Lelo, 2008; Lelo, 2011; Holy et al., 2013 ; Holy et al., 2014).

Parmi ceux-ci, Lelo (2008) déplore le mode de gestion des déchets à Kinshasa, il démontre les faiblesses des autorités et des populations kinoises en ce qui concerne la gestion rationnelle des ordures ménagères, il présente aussi les différents modes non hygiéniques d'évacuation des déchets à Kinshasa. Par ailleurs, il recommande que la gestion des déchets solides à Kinshasa s'effectue suivant la logique TRIVAC, c'est-àdire, Trier, Recycler, Incinérer, Valoriser, 
Communiquer comme cela se fait sous d'autres cieux. Holy et al. (2014) proposent quelques pistes de valorisation des déchets produits à Kinshasa, ils préconisent la subdivision de cette ville en zones fédérales de gestion des déchets et la création des sites potentiels des décharges finales.

\section{Conclusion}

L'enquête menée auprès des 30 producteurs agricoles (maraîchers, riz, maïs et manioc) et six agro-industriels (producteurs de café, bois scié, riz cargo et bière) dans cinq communes de Kinshasa (Lemba, Kinsenso, N'djili, Limété et Mont-Ngafula) a révélé que :

Au niveau de la production agricole, la paille de riz constitue le résidu végétal en surplus, tandis qu'il y a carence des matières végétales devant servir à la fertilisation en culture maraîchère. Les résidus issus du maraîchage causent probablement leur pollution au niveau des marchés et ménages consommateurs finaux. Ceci serait essentiellement dû à l'absence d'une politique efficace de gestion des déchets dans la ville de Kinshasa.

Les agro-industries enquêtées, produisent beaucoup de résidus végétaux mais ne procèdent pas rigoureusement à leur quantification. Ne pouvant les valoriser sur place, elles s'en débarrassent rapidement. Ces résidus sont alors vendus à vil prix, distribués gratuitement ou éliminés dans les cours d'eau les plus proches de leurs entreprises.

La présence de ces résidus agricoles et agroindustriels présente plusieurs impacts négatifs, principalement, il s'agit de l'encombrement de l'espace, la prolifération des mouches et des moustiques, l'apparition des maladies dues à l'insalubrité (malaria, fièvre typhoïde, etc.), l'apparition des odeurs nauséabondes et l'accumulation des flaques d'eau.

La production des déchets végétaux à Kinshasa est une conséquence normale des activités agricoles et agro-industrielles de la population kinoise. Leur bonne gestion implique la prise de conscience de la part des producteurs, des chercheurs et des décideurs politico-administratifs des potentialités de leur utilisation. La situation généralement rencontrée est la présence de ces résidus en excès et causant la pollution dans certains quartiers de la ville tandis que dans d'autres quartiers, il en manque. Dans ces derniers, ces résidus seraient utiles soit comme source énergétique, soit comme fertilisant organique. La résolution de la question de la pollution par les résidus végétaux, spécifiquement agricoles et agro-industriels, doit nécessairement passer par une stratégie comprenant d'abord leur quantification et caractérisation depuis leur production. Ensuite, il importe d'en déterminer les principaux usages possibles à l'état brut ou après une ou quelques transformations. Enfin, il faudrait en déterminer les besoins dans les sites où ces sous-produits peuvent être utilisés.

\section{Conflit d'intérêt}

Les auteurs de cet article déclarent qu'ils n'ont pas de conflits d'intérêts.

\section{Contribution des auteurs}

D-BBM et TMN se sont chargés de la planification de l'enquête et de la conception des fiches de questionnaires ; D-BBM et PKM ont réalisé l'enquête proprement dite auprès des agriculteurs et des agroindustriels, ensuite, ils ont transféré et analysé sur Excel les données récoltées ; La rédaction et la correction du manuscrit ont été effectuées par D-BBM, NKN et TMN.

\section{REFERENCES}

Amoo-Gottfried K, Hall DO. 1999. A biomass energy flow chart for Sierra Leone. Biomass and Bioenergy, 16: 361-376. DOI: http://dx.doi.org/doi:10.1016/ S0961-9534(99)00009-4

Balat M. 2011. Production of bioethanol from lignocellulosic materials via the biochemical pathway: A review. Energy Conversion and Management, 52: 858875. DOI: http://dx.doi.org/10.1016/j. 
enconman.2010.08.013

Binod P, Sindhu R, Singhania RR, Vikram S, Devi L, Nagalakshmi S, Kurien N, Sukumaran RK, Pandey A. 2010. Bioethanol production from rice straw: An overview. Bioresource Technology, 101(13): 4767-4774.

Carmen S. 2010. Cultivation of Pleurotus ostreatus and other edible mushrooms. Appl Microbiol Biotechnol., 85: 13211337. DOI: http://dx.doi.org/10.1007/ s00253-009-2343-7

Cooper CJ, Laing CA. 2007. A macro analysis of crop residue and animal wastes as a potential energy source in Africa. Journal of Energy in Southern Africa, 18(1): 10-19.

De Saint Moulin L, Kalombo TJL. 2005. Atlas de l'Organisation Administrative de la $R D C$. CEPAS : Kinshasa.

Demirbas AH, Demirbas I. 2007. Importance of rural bioenergy for developing countries. Energy Conversion and Management, 48(8): 2386-2398. DOI: http://dx.doi.org/10.1016/j.enconman.20 07.03.005

Ding SY, Himmel ME. 2006. The maize primary cell wall microfibril: a new model derived from direct visualization. J. Agric. Food Chem., 54: 597-606. DOI: $10.1021 / \mathrm{jf051851z}$

Hall DO, Rosillo-Calle F, Williams RH, Woods J. 1993. Biomass energy supply and prospects. In Renewable Energy: Sources for Fuel and Electricity, Johansson TB, Kelly H, Reddy AKN, Williams RH. (eds). Island Press, Washington DC; 593-651.

Holy HM, Nedeff V, Barsan N. 2013. Integrated management and sustainable waste Kinshasa and management of urban space. Troisième Colloque Francophone Pluridisciplinaire sur les Matériaux, l'Environnement et l'Electronique. Bacau (Roumanie).

Holy HM, Nedeff V, Kakese K, Barsan N, Mosnegutu E, Tomozei C. 2014. Municipal waste management in Limete,
Mont-Amba district of Kinshasa, Democratic Republic of the Congo. Journal of Engineering Studies and Research, 20(2): 39- 45.

Ingenosahel (Groupement d'Ingénieurs Conseil du Sahel). 1998. Etude de Faisabilité d'une Valorisation des Résidus Agricoles et Agro-industriels comme Combustibles Domestiques au Sénégal. Rapport de synthèse. Projet Sénégalo-Allemand Combustibles Domestiques.

Jingura RM, Matengaifa R. 2008. The potential for energy production from crop residues in Zimbabwe. Biomass and Bioenergy, 32: 1287-1292. DOI: http://dx.doi.org/ 10.1016/j. biombioe.2008.03.007

Jölli D, Giljum S. 2005. Unused Biomass extraction in Agriculture, Forestry and Fishery. SERI: Vienna, Austria.

Klass D. 2004. Biomass for Renewable Energy and Fuels. Encyclopedia of energy, Elsevier Inc.: New York.

Koopmans A, Koppejan J. 1997. Agricultural and forest residues, generation, utilization and availability. In Regional Consultation on Modern Applications of Biomass. FAO : Kuala Lumpur, Malasya.

Koyazibo AYF. 2012. Kinshasa. La pollution urbaine et ses impacts sur la santé humaine. Exposé présenté à l'occasion de la journée mondiale de l'environnement. CERSA BYBLOS, Kinshasa.

Lelo NF. 2008. Kinshasa, Ville \& Environnement. L'Harmattan : Paris.

Mulaji KC. 2011. Utilisation des Composts de Biodéchets Ménagers pour l'Amélioration de la Fertilité des Sols Acides de la Province de Kinshasa (République Démocratique du Congo). Thèse de Doctorat. Université de Liège Gembloux Agro-Bio Tech.

Lelo NF. 2011. Kinshasa, Planification \& Aménagement. L’Harmattan : Paris.

Müller HW, Trösch W. 1986. Screening of 
white-rot fungi for biological pretreatment of wheat straw for biogas production. Appl Microbiol Biotechnol., 24: 180-185 DOI: 10.1007/BF01982565

Murthy PS, Naidu MM. 2012. Sustainable management of coffee industry byproducts and value addition-A Review. Resources, Conservation and Recycling, 66: 45-58. DOI: http://dx.doi.org/ 10.1016/j.resconrec.2012.06.005

McKendry P. 2002. Energy production from biomass (part 1): overview of biomass. Review paper. Bioresource Technology, 83: 37-46. DOI: http://dx.doi.org/10. 1016/S0960-8524(01)00118-3

Singh J, Panesar BS, Sharma SK. 2008. Energy potential through agricultural biomass using geographical information system-A case study of Punjab. Biomass and Bioenergy, 32: 301 - 307. DOI : http://dx.doi.org/10.1016/j. biombioe.2007.10.003

Sivrikaya H, Peker H. 1999. Cultivation of
Pleurotus florida on Forest and Agricultural Wastes By Leaves of Tree and Wood Waste. Tr. J. Agriculture and Forestry, 23: 585-596. DOI : http://dx.doi.org/10.1007/s00253-0092343-7

Thiébaud S. 1995. Valorisation Chimique de Composés Lignocellulosiques : Obtention de Nouveaux Matériaux. Thèse de Doctorat. L'Institut National Polytechnique de Toulouse, p. 125.

Thioune L. 2009. Le biocharbon dans la politique énergétique du Sénégal. Programme pour la Promotion des Energie Renouvelables, de l'électrification rurale et de l'approvisionnement durable en combustibles domestiques. Vie $\mathrm{N}^{\circ} 11$ Mai - Juin, 1-12. Disponible sur www.peracod.sn 